Pacific Journal of Mathematics

EXTENSIONS OF SHEAVES OF ASSOCIATIVE ALGEBRAS BY 


\title{
EXTENSIONS OF SHEAVES OF ASSOCIATIVE ALGEBRAS BY NONTRIVIAL KERNELS
}

\author{
JoHN W. GRAY
}

Introduction. Let $X$ be a topological space, $A$ a sheaf of associative algebras over $X$ and $A$ a sheaf of two-sided $\Lambda$-modules considered as a sheaf of algebras with trivial multiplication. It was shown in [1] that the group $F(\Lambda, A)$ of equivalence classes of algebra extensions of $A$ with $A$ as kernel occurs naturally in an exact sequence

$$
\cdots \rightarrow H^{1}(X, A) \rightarrow F(\Lambda, A) \rightarrow \operatorname{Ext}^{2}(\Lambda, A) \rightarrow H^{2}(X, A) \rightarrow \cdots
$$

where $H^{*}(X, A)$ denotes the Cech cohomology of $X$ with coefficients in $A$. In this paper the same question will be discussed for the case in which $A$ has a non-trivial multiplication. It will be shown that under appropriate hypothese $F(\Lambda, A)$ occurs in a similar exact sequence, except that in the other terms of the sequence, $A$ must be replaced by the "bicenter" $K_{A}$ of $A$. A precise statement of the main result of this paper is given in Theorem 2. The methods used here are an adaptation of those used by S. MacLane in [2].

1. The extension problem. Let $R$ be a sheaf of rings on a topological space $X$. If $C$ and $D$ are sheaves of $R$-modules, then $H_{o m}$ $(C, D)$ will denote the sheaf of germs of $R$-homomorphisms of $C$ into $D$ and $\operatorname{Ext}_{R}^{n}(C, D)$ will denote the $n$th derived functor of $\operatorname{Hom}_{R}(C, D)$. If $A$ is a sheaf of associative $R$-algebras, then, as usual, $A^{*}$ will denote the opposite of $R$-algebras and $A^{e}=A \bigotimes_{R} A^{*}$ will denote the enveloping sheaf of $A$. $A$ is a sheaf of $A^{e}$-modules, the operation of $A^{e}$ on $A$ being given by the formula $\left(\lambda \otimes \mu^{*}\right)(\gamma)=\lambda \gamma \mu$.

$$
\text { Now, let } M_{A}^{\prime}=\operatorname{Hom}_{A *}(A, A) \oplus \operatorname{Hom}_{A}(A, A)
$$

where $\oplus$ denotes the direct sum. Then $M_{A}^{\prime}$, being the direct sum of sheaves of rings, is itself a sheaf of rings and $A$ can be considered as a sheaf of left and right $M_{A}^{\prime}$-modules as follows: Let $\sigma=\left(\sigma_{1}, \sigma_{2}\right) \in M_{A}^{\prime}$. Then the left action is given by $\sigma(\alpha)=\sigma_{1}(a)$ and the right action by $(a) \sigma=\sigma_{2}(a)$. Let

$$
M_{A}=\left\{\sigma \in M_{A}^{\prime} \mid a(\sigma b)=(a \sigma) b \text { for all } a, b \in A\right\} .
$$

Received June 15, 1960. This work has been supported by Office of Naval Research contract number Nonr. 266(57). 
Then $M_{A}$ is a subsheaf of subrings of $M_{A}^{\prime}$. $\quad M_{A}$ will be called the sheaf of germs of bimultiplications of $A$. Note that we cannot assert that $A$ is a sheaf of $M_{A}^{\rho}$-modules since we do not know that $(\sigma \alpha) \tau=\sigma(a \tau)$. If $\sigma$ and $\tau$ satisfy this relation then they are called permutable bimultiplications. The natural ring homomorphisms $A \rightarrow \operatorname{Hom}_{A *}(A, A)$ and $A \rightarrow$ $H_{0}(A, A)$ given respectively by left and right multiplication induce a ring homomorphism $\mu: A \rightarrow M_{A}$ whose image is a sheaf of two-sided ideals. The kernel $K_{A}$ of $\mu$ will be called the bicenter of $A$ and the cokernel $P_{A}$ of $\mu$ will be called the sheaf of germs of outer bimultiplications of $A . \quad P_{A}$ is a sheaf of rings and $K_{A}$ is a sheaf of left and right $P_{A}$-modules. As above, $K_{A}$ is not a sheaf of $P_{A}^{e}$-modules. Elements $\bar{\sigma}$ and $\bar{\tau}$ of $P_{A}$ such that $(\bar{\sigma} a) \bar{\tau}=\bar{\sigma}(a \bar{\tau})$ for all $a \in K_{A}$ will be called permutable. Note that $\bar{\sigma}$ and $\bar{\tau}$ are permutable if and only if representative elements $\sigma$ and $\tau$ in $M_{A}$ are also permutable.

An extension of a sheaf $A$ of $R$-algebras by a sheaf $A$ of $R$-algebras is an exact sequence.

$$
0 \rightarrow A \stackrel{i}{\longrightarrow} \Gamma \stackrel{p}{\longrightarrow} \Lambda \rightarrow 0
$$

of sheaves of $R$-algebras and $R$-algebra homomorphisms. As in [1], we shall say that such a sequence is locally trivial if there exists a covering $\mathscr{U}=\left\{U_{\alpha}\right\}$ of $X$ such that the restriction of the sequence to each $U_{\alpha}$ splits as an exact sequence of sheaves of $R$-modules. Hence if (1) is locally trivial then there exist $R$-module homomorphisms $j_{a}: A \mid U_{\alpha} \longrightarrow$ $\Gamma \mid U_{a}$ with $p j_{a}=$ identity. Furthermore, since $A$ is a sheaf of two-sided ideals in $\Gamma$, the map $\mu: A \longrightarrow M_{A}$ extends to a map $\mu_{\Gamma}: \Gamma \longrightarrow M_{\Delta}$. Thus, we may define the composition

$$
\theta_{\alpha}=(\operatorname{coker} \mu) \circ \mu_{\Gamma} \circ j_{\alpha}: \Lambda\left|U_{\alpha} \rightarrow P_{A}\right| U_{\alpha} .
$$

Since $\left(j_{\beta}-j_{\alpha}\right): \Lambda\left|U_{\alpha \beta} \longrightarrow A\right| U_{\alpha \beta}$, we see that $\theta_{\beta}=\theta_{\alpha}$ on $U_{\alpha \beta}=U_{\alpha} \cap U_{\beta}$. Hence $\left\{\theta_{\alpha}\right\}$ determines an element $\theta \in \operatorname{Hom}_{R}\left(\Lambda, P_{A}\right)$. We shall say that this $\theta$ is induced by the extension (1). Clearly $\theta$ is an algebra homomorphism whose image consists of permutable elements. Note that this implies that $K_{A}$ is a sheaf of $\Lambda^{e}$-modules via the operation of $P_{A}$ on $K_{A}$.

If $\theta \in \operatorname{Hom}_{R}\left(\Lambda, P_{A}\right)$ is an algebra homomorphism whose image consists of permutable elements, then, with respect to the usual equivalence relation, we wish to classify the extensions which induce $\theta$ in the manner described above.

2. The complexes. From [1], we recall that a sheaf $B$ of $R$ modules is said to be weakly $R$-projective if each stalk $B_{x}$ is an $R_{x^{-}}$ projective module and it is said to be $R$-coherent if there exists a covering $\mathscr{U}=\left\{U_{\alpha}\right\}$ such that for each $U_{\alpha}$ there are integers $p$ and $q$ and $R$-homomorphisms so that the sequence 


$$
R^{p}\left|U_{\alpha} \longrightarrow R^{q}\right| U_{\alpha} \longrightarrow B \mid U_{\alpha} \longrightarrow 0
$$

is exact. Also, as in [1], $C^{*}(X, B)$ will denote the direct limit over coverings $\mathscr{U}$ indexed by $X$ of the Cech cohomology complexes $C^{*}(\mathscr{C}, B)$. If $S_{0}(\Lambda)=R$ and $S_{n}(\Lambda), n>0$ denotes the $n$-fold tensor product of $\Lambda$ with itself, then we define

$$
L^{i, j}(B)=C^{i}\left(X, \operatorname{Hom}_{R}\left(S_{\jmath}(\Lambda), B\right)\right) .
$$

Proposition 1. If $X$ is paracompact Hausdorff and if $A$ is weakly $R$-projective and $R$-coherent, then, for each $n \geqq 0$,

$$
0 \longrightarrow L^{*, n}\left(K_{A}\right) \longrightarrow L^{*, n}(A) \stackrel{\mu *}{\longrightarrow} L^{*, n}\left(M_{A}\right) \stackrel{\pi *}{\longrightarrow} L^{*, n}\left(P_{A}\right) \longrightarrow 0
$$

is an exact sequence of complexes, the mappings being those induced by the exact sequence of sheaves

$$
0 \longrightarrow K_{A} \longrightarrow A \stackrel{\mu}{\longrightarrow} M_{A} \stackrel{\pi}{\longrightarrow} P_{A} \longrightarrow 0
$$

Proof. In [1] it was shown that if $\Lambda$ is weakly $R$-projective and $R$-coherent then so is $S_{n}(\Lambda)$ and hence the sheaves $\operatorname{Ext}_{R}^{i}\left(S_{n}(\Lambda), B\right)=0$ for $i>0, n \geqq 0$ and for all $B$. Hence, for each $n \geqq 0$, there is an exact sequence of sheaves

$$
\begin{gathered}
0 \longrightarrow \operatorname{Hom}_{R}\left(S_{n}(\Lambda), K_{A}\right) \longrightarrow \operatorname{Hom}_{R}\left(S_{n}(\Lambda), A\right) \longrightarrow \operatorname{Hom}_{R}\left(S_{n}(\Lambda), M_{A}\right) \\
\longrightarrow \operatorname{Hom}_{R}\left(S_{n}(\Lambda), P_{A}\right) \longrightarrow 0 .
\end{gathered}
$$

If $X$ is paracompact Hausdorff then $C^{*}(X,-)$ is an exact functor and hence we get the indicated sequence of complexes.

We would like to consider each of the complexes $L^{i .9}(-)$ in the preceding proposition as a bicomplex in some manner which reflects a given structure of $K_{A}$ as a sheaf of $\Lambda^{e}$-modules and which coincides with the usual structure of $\operatorname{Hom}_{R}\left(S_{n}(A),-\right)$ as a complex. This is too much to ask, but such a structure on $L^{i, j}(A)$ can be approximated as follows: Let $\theta \in \operatorname{Hom}\left(\Lambda, P_{A}\right)$ be an algebra homomorphism whose image consists of permutable elements. If $\theta$ is regarded as an element of $L^{0,1}\left(P_{A}\right)$, then by exactness there is an element $\sigma \in L^{0,1}\left(M_{A}\right)$ such that $\pi_{*}(\sigma)=\theta$. Let $\sigma$ be represented by cocycle $\left\{\sigma_{\alpha}\right\}$ on some sufficiently fine covering $\mathscr{Z}$. Given this date, we can define a "coboundary" operator $\delta_{\sigma}$ on $L^{m n}(A)$ by the following formula. Let $k \in L^{m, n}(A)$ be represented by a cochain $\left\{k_{\alpha_{1}, \ldots, \alpha_{m}}\right\}$ on $\mathscr{\ell}$. Then

$$
\begin{gathered}
\delta_{\sigma} k_{\alpha_{0}, \ldots, \alpha_{m}}\left(\lambda_{1, \ldots,} \lambda_{n+1}\right)=\sigma_{\alpha_{0}}\left(\lambda_{1}\right) k_{\alpha_{0}, \ldots, \alpha_{m}}\left(\lambda_{2, \ldots,} \lambda_{n+1}\right) \\
+\sum_{i=1}^{n}(-1)^{i} k_{\alpha_{0}, \ldots, \alpha_{m}}\left(\lambda_{1, \ldots}, \lambda_{i} \lambda_{i+1, \ldots,}, \lambda_{n+1}\right) \\
+(-1)^{n+1} k_{\alpha_{0}, \ldots, \alpha_{m}}\left(\lambda_{1, \ldots,}, \lambda_{n}\right) \sigma_{\alpha_{0}}\left(\lambda_{n+1}\right) .
\end{gathered}
$$


We shall see that the restriction of $\delta_{\sigma}$ to $L^{i, j}\left(K_{A}\right)$ is in fact a good coboundary operator.

In order to investigate the properties of $\delta_{\sigma}$ and the relations between $\delta_{\sigma}$ and the Cech coboundary operator $\hat{\delta}$, we must introduce some more notation.

(2.1) To avoid constantly writing variables we make the following convention: If $r$ is a function of $p$ variables and $s$ is a function of $q$ variables, both with values in an algebra, then $r \cdot s$ is the function of $p+q$ variables defined by

$$
r \cdot s\left(\lambda_{1, \ldots}, \lambda_{p+q}\right)=r\left(\lambda_{1, \ldots,} \lambda_{p}\right) \cdot s\left(\lambda_{p+1, \ldots,} \lambda_{p+q}\right) .
$$

(2.2) $m$ will denote ambiguously the multiplication in all of the algebras which appear here.

(2.3) Since $\theta$ is an algebra homomorphism, $\pi_{*}\left(\sigma_{\alpha} \cdot \sigma_{\alpha}-\sigma_{\alpha} \circ m\right)=0$ Hence there exists an $f \in L^{0.2}(A)$ which is represented by a cochain $\left\{f_{\alpha}\right\}$ on $\mathscr{U}$ such that

$$
\mu_{*} f_{\alpha}=\sigma_{\alpha} \cdot \sigma_{\alpha}-\sigma_{\alpha} \circ m \text {. }
$$

(2.4) Since $\pi_{*}(\hat{\delta} \sigma)=\hat{\delta} \pi_{*}(\sigma)=0$, there exists an $h \in L^{1,1}(A)$ which is represented by a cochain $\left\{h_{\alpha \beta}\right\}$ on $\mathscr{Q}$ such that

$$
\mu_{*} h_{\alpha \beta}=(\hat{\delta} \sigma)_{\alpha \beta} .
$$

(2.5) If $\sigma^{\prime} \in L^{0.1}\left(M_{A}\right)$ also satisfies $\pi_{*}\left(\sigma^{\prime}\right)=\theta$, then $\pi_{*}\left(\sigma^{\prime}-\sigma\right)=0$ and hence there exists a $\bar{\sigma} \in L^{0.1}(A)$ which is represented by a cochain $\left\{\bar{\sigma}_{\alpha}\right\}$ on $\mathscr{U}$ such that

$$
\mu_{*} \bar{\sigma}_{\alpha}=\bar{\sigma}_{\alpha}^{\prime}-\bar{\sigma}_{\alpha} .
$$

Using these notations the following result in easily checked:

Proposition 2. If $k \in L^{m . n}(A)$ is represented by $\left\{k_{\alpha_{0}, \ldots, \alpha_{m}}\right\}$ on $\mathscr{U}$, then

$$
\begin{aligned}
\delta_{\sigma} \delta_{\sigma} k_{\alpha_{0}, \ldots, \alpha_{m}}= & f_{\alpha_{0}} \cdot k_{\alpha_{0}, \ldots, \alpha_{m}}-k_{\alpha_{0}, \ldots, \alpha_{m}} \cdot f_{\alpha_{0}} \\
\delta_{\sigma}(\widehat{\delta} k)_{\alpha_{0}, \ldots, \alpha_{m+1}}= & \left(\widehat{\delta} \delta_{\sigma} k\right)_{\alpha_{0}, \ldots, \alpha_{m+1}}-h_{\alpha_{0}, \alpha_{1}} k_{\alpha_{1}, \ldots, \alpha_{m+1}} \\
& -(-1)^{n+1} k_{\alpha_{1}, \ldots, \alpha_{m+1}} h_{\alpha_{0}, \alpha_{1}}
\end{aligned}
$$

$$
\delta_{\sigma^{\prime}} k_{\alpha_{0}, \ldots, \alpha_{m}}=\delta_{\sigma} k_{\alpha_{0}, \ldots, \alpha_{m}}+\bar{\sigma}_{\alpha_{0}} k_{\alpha_{0}, \ldots, \alpha_{m}}+(-1)^{n+1} k_{\alpha_{0}, \ldots, \alpha_{m}} \bar{\sigma}_{\alpha_{0}} \text {. }
$$

COROLlaRy. $L^{i, j}\left(K_{A}\right)$ is a bicomplex with respect to the pair of differential operators $\hat{\delta}, \delta_{\sigma}$. The total differential operator is given by 


$$
\delta=(-1)^{j+1} \widehat{\delta}+\delta_{\sigma} .
$$

This differential operator depends only on $\theta$.

Finally, we shall need to know something about the behavior of $\hat{\delta}$ on products of low dimensional cochains, where Cech cochains are multiplied by multiplying the values (suitably restricted when necessary) on corresponding elements of the nerve of a covering according to the convention of 2.1. It is easy to verify the following statements by explicit calculation.

Proposition 3. If $r \in L^{0, p}(A)$ and $s \in L^{0, q}(A)$ are represented on $\mathscr{U}$ by $\left\{r_{\alpha}\right\}$ and $\left\{s_{\alpha}\right\}$ respectively, then $\hat{\delta}(r \cdot s) \in L^{1, p+q}(A)$ and

$$
\hat{\delta}(r \cdot s)_{\alpha \beta}=(\hat{\delta} r)_{\alpha \beta} \cdot s_{\alpha}+r_{\alpha} \cdot(\hat{\delta} s)_{\alpha \beta}+(\hat{\delta} r)_{\alpha \beta} \cdot(\delta s)_{\alpha \beta} \text {. }
$$

If $t \in L^{1, p}(A)$ and $u \in L^{1, q}(A)$ are represented on $\mathscr{U}$ by $\left\{t_{\alpha \beta}\right\}$ and $\left\{u_{\alpha \beta}\right\}$ respectively then $\hat{\delta}(t \cdot u) \in L^{2, p+q}(A)$ and

$$
\begin{gathered}
(\hat{\delta}(t \cdot u))_{\alpha \beta \gamma}=(\hat{\delta} t)_{\alpha \beta \gamma} \cdot u_{\alpha \gamma}+t_{\alpha \gamma} \cdot(\hat{\delta} u)_{\alpha \beta \gamma}+(\hat{\delta} t)_{\alpha \beta \gamma} \cdot(\hat{\delta} u)_{\alpha \beta \gamma}-t_{\alpha \beta} \cdot u_{\beta \gamma} \\
-t_{\beta \gamma} \cdot u_{\alpha \beta} .
\end{gathered}
$$

Finally, if $r \in L^{m, p}(A)$ and $s \in L^{m, q}(A)$ then $\delta_{\sigma}$ satisfies the good coboundary formula.

$$
\delta_{\sigma}(r \cdot s)=\left(\delta_{\sigma} r\right) \cdot s+(-1)^{p} r \cdot \delta_{\sigma} s
$$

3. The obstruction. We shall regard the complex $L^{i, j}\left(K_{A}\right)$ as being filtered by the second degree and we define $F^{p}(L)=\sum_{j \geq p} L^{i, j}\left(K_{A}\right)$. In analogy with the proceedings of [1], the classical results for extensions of algebras suggest that each algebra homomorphism $\theta \in \operatorname{Hom}(\Lambda$, $P_{A}$ ) whose range consists of permutable elements determines an "obstruction" in $H^{3}\left(F^{1}(L)\right)$; this obstruction being zero if and only if there exists an extension which induces $\theta$ in the manner described in $\S 1$. A representative cocycle for such a cohomology class would be an element of $L^{2,1}\left(K_{A}\right) \oplus L^{1,2}\left(K_{A}\right) \oplus L^{0,3}\left(K_{A}\right)$.

$$
\text { Let } \sigma \in L^{0,1}(A) \text { satisfy } \pi_{*} \sigma=\theta \text { and let }
$$

$f \in L^{0.2}(A)$ and $h \in L^{1,1}(A)$ be defined as in 2.3 and 2.4. Then the components of a representative cocycle of the "obstruction" to $\theta$ are defined as follows:

(i) Since $\mu_{*}(\hat{\delta} h)=\widehat{\delta} \mu_{*} h=0$, there exists an element $a \in L^{2,1}\left(K_{4}\right)$ which is represented by a cochain $\left\{a_{\alpha \beta \gamma}\right\}$ on $\mathscr{U}$ such that

$$
a_{\alpha \beta \gamma}=(\widehat{\delta} h)_{\alpha \beta \gamma}
$$

(ii) A standard elementary calculation shows that $\mu_{*}\left(\delta_{\sigma} f\right)=0$. 
Hence there exists an element $c \in L^{03}\left(K_{A}\right)$ which is represented by a cochain $\left\{c_{\alpha}\right\}$ on $\mathscr{U}$ such that $c_{\alpha}=\delta_{\sigma} f_{\alpha}$.

(iii) An equally elementary calculation shows that $\mu_{*}\left[\hat{\delta} f-\delta_{\sigma} h-\right.$ $h \cdot h]=0$. Hence there exists an element $b \in L^{1,2}\left(K_{A}\right)$ which is represented by a cochain $\left\{b_{\alpha \beta}\right\}$ on $\mathscr{U}$ such that

$$
b_{\alpha \beta}=-(\hat{\delta} f)_{\alpha \beta}+\delta_{\sigma} h_{\alpha \beta}+h_{\alpha \beta} \cdot h_{\alpha \beta}
$$

THEOREM 1. Let's $s=a \oplus b \oplus c$. Then $s$ is a cocycle of $F^{1}(L)$ whose cohomology class depends only on $\theta$.

Definition. The cohomology class of $s$ will be denoted by $O b(\theta)$ and will be called the obstruction to $\theta$.

THEOREM 2. Let $X$ be paracompact Hausdorff and let $A$ be weakly $R$-projective and $R$-coherent. Then $O b(\theta)=0$ if and only if there is an extension of $\Lambda$ by $A$ which induces $\theta$. If $O B(\theta)=0$, then the set $F_{\theta}(\Lambda, A)$ of equivalence classes of extensions which induce $\theta$ is in oneto-one correspondence with the set of elements of the group $H^{2}\left(F^{1} K\right)$, and hence the following two sequences are exact.

(1) $0 \longrightarrow H^{1}\left[\operatorname{Hom}_{R}\left(S_{*}(\Lambda), K_{A}\right)\right] \longrightarrow \operatorname{Ext}_{A^{e}}^{1}\left(\Lambda, K_{A}\right) \longrightarrow H^{1}\left(X, K_{A}\right)$

( 2$) \quad 0 \longrightarrow H^{2}\left[\operatorname{Hom}_{R}\left(S_{*}(\Lambda), K_{A}\right)\right] \longrightarrow F_{\theta}(\Lambda, A) \longrightarrow$ $H^{1}\left(X, \operatorname{Hom}_{R}\left(\Lambda, K_{A}\right)\right) \longrightarrow$

Proof of Theorem 1. It is clear that $\hat{\delta} a=\hat{\delta} \widehat{\delta} h=0$, and, by 2.6, that $\delta_{\sigma} c=\delta_{\sigma} \delta_{\sigma} f=0$. Thus, to prove that $s$ is a cocycle we must show that $\hat{\delta} b=\delta_{\sigma} a$ and that $\hat{\delta} c=-\delta_{\sigma} b$. To derive the first expression, we have by definition that

$$
(\hat{\delta} b)_{\alpha \beta \gamma}=-(\hat{\delta} \hat{\delta} f)_{\alpha \beta \gamma}+\left(\hat{\delta} \delta_{\sigma} h\right)_{\alpha \beta \gamma}+\hat{\delta}(h \cdot h)_{\alpha \beta \gamma}
$$

The first term is zero and the second and third terms can be expanded by 2.7 and 2.10 respectively. After obvious cancellations, this yields

$$
(\hat{\delta} b)_{\alpha \beta \gamma}=\delta_{\sigma}(\hat{\delta} h)_{\alpha \beta \gamma}+(\hat{\delta} h)_{\alpha \beta \gamma} \cdot h_{\alpha \gamma}+h_{\alpha \gamma} \cdot(\hat{\delta} h)_{\alpha \beta \gamma}+(\hat{\delta} h)_{\alpha \beta \gamma} \cdot(\hat{\delta} h)_{\alpha \beta \gamma}
$$

Since $\hat{\delta} h=a \in L^{2,1}\left(K_{4}\right)$, on a sufficiently fine covering multiplication by $(\hat{\delta} h)_{\alpha \beta \gamma}$ is zero and hence $\hat{\delta} b=\delta_{\sigma} a$. Similarly, since $c=\delta_{\sigma} f, \hat{\delta} c$ can be expanded by the equation, 2.7, for commuting $\hat{\delta}$ and $\delta_{\sigma}$. The resulting expression can be simplified by using equations 2.6 and 2.11 and the definition of $b$ in (ii). This yields easily that

$$
(\hat{\delta} c)_{\alpha \beta}=\delta_{\sigma}\left[(\hat{\delta} f)_{\alpha \beta}-\delta_{\sigma} h_{\alpha \beta}-h_{\alpha \beta} \cdot h_{\alpha \beta}\right]=-\delta_{\sigma} b_{\alpha \beta} .
$$

Thus $s$ is a cocycle. 
The definition of $s$ depends on the choices of $b, h$, and $f$. We shall show that changing any of these changes $s$ by a coboundary and that any cocycle cohomologous to $s$ can be obtained by such a choice.

Suppose that $h^{\prime}$ satisfies $\mu_{*} h^{\prime}=\hat{\delta} \sigma$ and $f^{\prime}$ satisfies $\mu_{*} f^{\prime}=\sigma \cdot \sigma-\sigma \circ m$. Then $h^{\prime}-h=\bar{h} \in L^{1,1}\left(K_{A}\right)$ and $f^{\prime}-f=\bar{f} \in L^{0,2}\left(K_{A}\right)$. If $s^{\prime}$ denotes the cocycle corresponding to $\sigma, h^{\prime}$ and $f^{\prime}$, then it is easy to see that

$$
s^{\prime}-s=\left(\hat{\delta}+\delta_{\sigma}\right) \bar{h}+\left(-\hat{\delta}+\delta_{\sigma}\right) \bar{f}=\delta(\bar{h}+\bar{f}) .
$$

Conversely, if $\bar{h} \oplus \bar{f}$ is any 2-cochain of $F^{1}(L)$, then $h+\bar{h}$ and $f+\bar{f}$ are admissable liftings of $\hat{\delta}_{\sigma}$ and $\sigma \cdot \sigma-\sigma \circ m$ respectively and this change alters $s$ by $\delta(\bar{h} \oplus \bar{f})$. Hence, in this manner we obtain all cocycles cohomologous to $s$.

It remains to show that if $\pi_{*} \sigma^{\prime}=\theta$, then $h^{\prime}$ and $f^{\prime}$ can be chosen so that the corresponding cocycle $s^{\prime}=a^{\prime}+b^{\prime}+c^{\prime}=s$. Since $\pi_{*}\left(\sigma^{\prime}\right.$. $-\sigma)=0$, there is a $\bar{\sigma} \in L^{0,1}(A)$ such that $\mu_{*} \bar{\sigma}=\sigma^{\prime}-\sigma$. Let $h^{\prime}=h+$ $\hat{\delta} \bar{\sigma}$ and $f^{\prime}=f+\delta_{\sigma} \bar{\sigma}+\bar{\sigma} \cdot \bar{\sigma}$. Then it is immediate that $h^{\prime}$ and $f^{\prime}$ are liftings of $\hat{\delta} \sigma^{\prime}$ and $\sigma^{\prime} \cdot \sigma^{\prime}-\sigma^{\prime} \cdot m$ respectively and that $a^{\prime}=\hat{\delta} h^{\prime}=a$. The difference $\delta_{\sigma^{\prime}} f^{\prime}-\delta_{\sigma} f$ can be expressed by 2.8. Using 2.6 and 2.11, it is easily seen that this difference is zero and hence $c^{\prime}=c$. The only difficult point is to show that $b^{\prime}=b$. By definition

$$
b^{\prime}=-\widehat{\delta} f^{\prime}+\delta_{\sigma^{\prime}} h^{\prime}+h^{\prime} \cdot h^{\prime}
$$

Using the definitions of $f^{\prime}$ and $h^{\prime}$ and rearranging terms, we arrive at the equality

$$
\begin{aligned}
b_{\alpha \beta}{ }^{\prime}-b_{\alpha \beta}= & {\left[\delta_{\sigma} \hat{\delta} \bar{\sigma}_{\alpha \beta}-\hat{\delta} \delta_{\sigma} \bar{\sigma}_{\alpha \beta}\right]+\left[\bar{\sigma}_{\alpha} \cdot h_{\alpha \beta}+h_{\alpha \beta} \cdot \bar{\sigma}_{\alpha}+\widehat{\delta} \bar{\sigma}_{\sigma \beta} \cdot h_{\alpha \beta}+h_{\alpha \beta} \cdot \hat{\delta} \bar{\sigma}_{\alpha \beta}\right] } \\
& +\left[\bar{\sigma}_{\alpha} \cdot \hat{\delta} \bar{\sigma}_{\alpha \beta}+\widehat{\delta} \bar{\sigma}_{\alpha \beta} \cdot \bar{\sigma}_{\alpha}+\hat{\delta} \bar{\sigma}_{\alpha \beta} \cdot \hat{\delta} \bar{\sigma}_{\alpha \beta}-\hat{\delta}(\bar{\sigma} \cdot \bar{\sigma})_{\alpha \beta}\right] .
\end{aligned}
$$

The third bracket is zero by the formula 2.9 for the Cech coboundary of a product and the first bracket equals $-h_{\alpha \beta} \cdot \bar{\sigma}_{\beta}-\bar{\sigma}_{\beta} \cdot h_{\alpha \beta}$ by the rule 2.7 for interchanging $\hat{\delta}_{\sigma}$ and $\delta$. Hence the sum of the first two brackets is zero and therefore $b^{\prime}=b$.

\section{Proof of Theorem 2. Suppose $0 \longrightarrow A \stackrel{i}{\longrightarrow} \Gamma \stackrel{p}{\longrightarrow} A \longrightarrow 0$ is an} extension. By Proposition 3.1 of [1], the hypotheses imply that any such extension is locally trivial considered as an extension of sheaves of $R$-modules. Hence there exists a covering $\mathscr{U}=\left\{U_{\alpha}\right\}$ which carries $R$ module homomorphisms $j_{\alpha} \cdot \Lambda\left|U_{a} \longrightarrow \Gamma\right| U_{a}$ with $p \cdot j_{\alpha}=$ identity. If $\sigma_{\alpha}: \Lambda\left|U_{\alpha} \rightarrow M_{A}\right| U_{\alpha}$ is defined by $\left[\sigma_{\alpha}(\lambda)\right](a)=j_{\alpha}(\lambda) \cdot a$ and $(a)\left[\sigma_{\alpha}(\lambda)\right]=$ $a \cdot j_{\alpha}(\lambda)$ then $\left\{\sigma_{\alpha}\right\}$ determines an element $\sigma \in L^{0,1}\left(M_{A}\right)$ which is a lifting of the homomorphism $\theta$ induced as in $\S 1$ by the given extension. If we define $h_{\alpha \beta}=j_{\beta}-j_{\alpha}$ and $f_{\alpha}=j_{\alpha} j_{\alpha}-j_{\alpha} \circ m$, then the corresponding elements $h \in L^{1,1}(A)$ and $f \in L^{0, \hat{2}}(A)$ satisfy $\mu_{*} h=\delta \sigma$ and $\mu_{*} f=\sigma \cdot \sigma-$ 
$\sigma \circ m$. Elementary calculations show that for this choice of $h$ and $f$ we get that $s=a \oplus b \oplus c=0$ and hence $O b(\theta)=0$.

Conversely, if $\operatorname{Ob}(\theta)=0$, then on some sufficiently fine covering $\mathscr{U}$, we may choose $\left\{f_{\alpha}\right\} \in \widehat{C}^{0}\left(\mathscr{U}, \operatorname{Hom}_{R}\left(S_{2}(\Lambda), A\right)\right)$ and $\left\{h_{\alpha \beta}\right\} \in \widehat{C}^{1}\left(U, \operatorname{Hom}_{R}(\Lambda, A)\right)$ so that $\delta_{\sigma} f_{\alpha}=0,(\hat{\delta} h)_{\alpha \beta \gamma}=0$ and $(\hat{\delta} f)_{\alpha \beta}=\delta_{\sigma} h_{\alpha \beta}+h_{\alpha \beta} \cdot h_{\alpha \beta}$. As in [1], we define $\Gamma$ to be the sheaf which is the quotient of $\mathrm{U}_{a}(A \oplus \Lambda) \mid U_{\alpha}$ by the relation

$$
\left(a+h_{\alpha \beta}(\lambda), \lambda\right)_{\alpha} \sim(a, \lambda)_{\beta} \text { for }(a, \lambda) \in A \oplus \Lambda \mid U_{\alpha \beta} .
$$

Multiplication in $\Gamma$ is given by the formula

$$
(a, \lambda)_{\alpha} \cdot\left(a^{\prime}, \lambda^{\prime}\right)_{\alpha}=\left(a a^{\prime}+\sigma_{\alpha}(\lambda) a^{\prime}+a \sigma_{\alpha}(\lambda)+f_{\alpha}\left(\lambda, \lambda^{\prime}\right), \lambda \lambda^{\prime}\right)_{\alpha} .
$$

It is easy to show that this multiplication is associative since $\delta_{\sigma} f=0$ and that it agrees with the equivalence relation since $\widehat{\delta} f=\delta_{\sigma} h+h \cdot h$.

It follows then, exactly as in MacLane [2] that the set of equivalence classes of extensions which realize a given $\theta$ with $O b(\theta)=0$ is in one-toone correspondence with the set of elements of the group $H^{2}\left(F^{1}(L)\right)$. The exact sequences are derived exactly as in [1] from the exact sequences of complexes

and

$$
\begin{aligned}
& 0 \longrightarrow F^{1} L \longrightarrow F^{0} L \longrightarrow E_{0}^{*, 0} \longrightarrow 0 \\
& 0 \longrightarrow F^{2} L \longrightarrow F^{1} L \longrightarrow E_{0}^{*, 1} \longrightarrow 0
\end{aligned}
$$

4. Examples. (1) If $K_{A}=0$ then all obstructions are zero and all terms involving $K_{A}$ in the exact sequence containing $F_{\theta}(\Lambda, A)$ are zero. Hence there is a unique extension of $\Lambda$ by $A$ which induces a given $\theta \in \operatorname{Hom}_{R}\left(\Lambda, P_{A}\right)$. As in MacLane [2], this extension can be described as the "graph" of $\theta$; i.e., the pull-back of the pair of maps $\theta$ : $\Lambda \longrightarrow P_{A}, \pi: M_{A} \longrightarrow P_{A}$.

(2) If $K_{A}=A$, then the map $\mu: A \longrightarrow M_{A}$ is the zero map and hence $M_{A}=P_{A}$. Consequently, if $\theta \in \operatorname{Hom}_{R}\left(\Lambda, P_{A}\right)$ is given, then $\sigma$ may be chosen equal to $\theta$ and so $\delta \sigma$ and $\sigma \cdot \sigma-\sigma \circ m$ are both zero. Therefore, any cocycle $f \oplus h \in L^{0,2}(A) \oplus L^{1,1}(A)$ is a lifting of these two terms. It follows that $O b(\theta)=0$ and that $F_{\theta}(\Lambda, A)=H^{2}\left(F^{1} L\right)$. Thus the results of [1] are a special case of the results of this paper.

(3) We wish to discuss more thoroughly a remark in $\S 3.3$ of [1]. Let $X$ be paracompact Hausdorff and let $\Lambda$ be a weakly $R$-projective and $R$-coherent sheaf of $R$-algebras. Suppose that $A$ is a sheaf of $R$ algebras and that

$$
0 \longrightarrow A \longrightarrow \Gamma \longrightarrow \Lambda \longrightarrow 0
$$

is an exact sequence of $R$-modules. Let $\mathscr{U}=\left\{U_{\alpha}\right\}$ be a sufficiently fine covering of $X$ and let $\left\{j_{\alpha}\right\} \in \widehat{C}^{0}\left(\mathscr{U}, \operatorname{Hom}_{R}(\Lambda, \Gamma)\right)$ determine the locally 
trivial structure of $\Gamma$ and let $h_{\alpha \beta}=(\hat{\delta} j)_{\alpha \beta}$. An algebra homomorphism $\theta \in \operatorname{Hom}_{R}\left(\Lambda, P_{A}\right)$ whose image consists of permutable elements will be called compatible with the locally trivial structure of $\Gamma$ if there exists a lifting $\sigma \in L^{0,1}\left(M_{A}\right)$ of $\theta$ which is represented by a cochain $\left\{\sigma_{\alpha}\right\}$ on $\mathbb{Z}$ such that $\mu_{*} h=\widehat{\delta} \sigma$. Furthermore, an element $f \in L^{0,2}(A)$ will be called a multiplication compatible with $\theta$ and $h$ if $\mu_{*} f=\sigma \cdot \sigma-\sigma \circ m, \widehat{\delta} f=$ $\delta_{\sigma} h+h \cdot h$ and $\delta_{\sigma} f=0$. The set of equivalence classes with respect to the usual equivalence relation of such multiplications will be denoted by $F_{\theta, h}(A, A)$. We wish to calculate $F_{\theta, h}(A, A)$.

Proceeding as in $\S 2$, let $f \in L^{0,2}(A)$ be a cochain such that $\mu_{*} f=$ $\sigma \cdot \sigma-\sigma \circ m$. Corresponding to $f \oplus h$ there is an obstruction cocycle $s(h)=c \oplus b \oplus 0$. The only relevant changes of $s(h)$ are given by varying $f$ by an element $\bar{f} \in L^{0,2}\left(K_{A}\right)$. Such a change alters $s$ by a coboundary in $F^{2} L$. Hence we obtain the result:

THEOREM. Corresponding to $\theta$ and $h$, there is an obstruction cohomology class $\operatorname{Ob}(\theta, h) \in H^{3}\left(F^{2} L\right)$ which is zero if and only if there exists a multiplication compatible with $\theta$ and $h$. If $O b(\theta, h)=0$ then $F_{\theta, h}(\Lambda, A)$ is in one-to-one correspondence with the elements of the group $H^{2}\left[\operatorname{Hom}_{R}\left(S_{*}(\Lambda), K_{A}\right)\right]$.

\section{BIBLIOGRAPHY}

1. J. W. Gray, Extensions of Sheaves of Algebras, Ill. J. of Math., 5 (1961), 159-174.

2. Saunders MacLane, Extensions and obstructions for rings, Ill. J. of Math., 2 (1958), 316-345.

COLUMBIA UNIVERSITY 



\title{
PACIFIC JOURNAL OF MATHEMATICS
}

\section{EDITORS}

\author{
Ralph S. Phillips \\ Stanford University \\ Stanford, California \\ F. H. BrownelL \\ University of Washington \\ Seattle 5 , Washington
}

A. L. Whiteman

University of Southern California

Los Angeles 7. California

L. J. PAIGE

University of California

Los Angeles 24, California

\section{ASSOCIATE EDITORS}

\author{
E. F. BECKENBACH \\ T. M. CHERRY
} \\ D. DERRY \\ M. OHTSUKA \\ UNIVERSITY OF BRITISH COLUMBIA \\ CALIFORNIA INSTITUTE OF TECHNOLOGY \\ UNIVERSITY OF CALIFORNIA \\ MONTANA STATE UNIVERSITY \\ UNIVERSITY OF NEVADA \\ NEW MEXICO STATE UNIVERSITY \\ OREGON STATE COLLEGE \\ UNIVERSITY OF OREGON \\ OSAKA UNIVERSITY \\ UNIVERSITY OF SOUTHERN CALIFORNIA
}

H. L. ROYDEN

E. SPANIER
E. G. STRAUS

F. WOLF

\section{SUPPORTING INSTITUTIONS}

\author{
STANFORD UNIVERSITY \\ UNIVERSITY OF TOKYO \\ UNIVERSITY OF UTAH \\ WASHINGTON STATE COLLEGE \\ UNIVERSITY OF WASHINGTON \\ AMERICAN MATHEMATICAL SOCIETY \\ CALIFORNIA RESEARCH CORPORATION \\ HUGHES AIRCRAFT COMPANY \\ SPACE TECHNOLOGY LABORATORIES \\ NAVAL ORDNANCE TEST STATION
}

Mathematical papers intended for publication in the Pacific Journal of Mathematics should be typewritten (double spaced), and the author should keep a complete copy. Manuscripts may be sent to any one of the four editors. All other communications to the editors should be addressed to the managing editor, L. J. Paige at the University of California, Los Angeles 24, California.

50 reprints per author of each article are furnished free of charge; additional copies may be obtained at cost in multiples of 50 .

The Pacific Journal of Mathematics is published quarterly, in March, June, September, and December. The price per volume (4 numbers) is $\$ 12.00$; single issues, $\$ 3.50$. Back numbers are available. Special price to individual faculty members of supporting institutions and to individual members of the American Mathematical Society: $\$ 4.00$ per volume; single issues, $\$ 1.25$.

Subscriptions, orders for back numbers, and changes of address should be sent to Pacific Journal of Mathematics, 103 Highland Boulevard, Berkeley 8, California.

Printed at Kokusai Bunken Insatsusha (International Academic Printing Co., Ltd.), No. 6 , 2-chome, Fujimi-cho, Chiyoda-ku, Tokyo, Japan.

PUBLISHED BY PACIFIC JOURNAL OF MATHEMATICS, A NON-PROFIT CORPORATION

The Supporting Institutions listed above contribute to the cost of publication of this Journal, but they are not owners or publishers and have no responsibility for its content or policies. 


\section{Pacific Journal of Mathematics}

\section{Vol. 11, No. $3 \quad$ BadMonth, 1961}

Errett Albert Bishop, A generalization of the Stone-Weierstrass theorem ..........

Hugh D. Brunk, Best fit to a random variable by a random variable measurable with

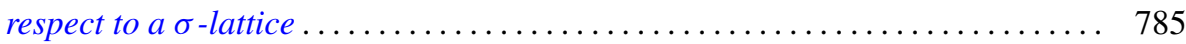

D. S. Carter, Existence of a class of steady plane gravity flows . . . . . . . . . . 803

Frank Sydney Cater, On the theory of spatial invariants ............... 821

S. Chowla, Marguerite Elizabeth Dunton and Donald John Lewis, Linear

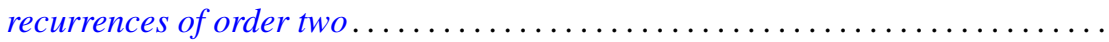

Paul Civin and Bertram Yood, The second conjugate space of a Banach algebra as

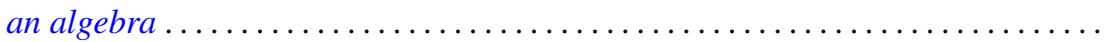

William J. Coles, Wirtinger-type integral inequalities .

Shaul Foguel, Strongly continuous Markov processes ....................

David James Foulis, Conditions for the modularity of an orthomodular lattice ...... Jerzy Górski, The Sochocki-Plemelj formula for the functions of two complex variables.

John Walker Gray, Extensions of sheaves of associative algebras by non-trivial

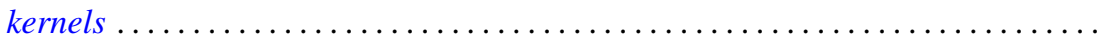

Maurice Hanan, Oscillation criteria for third-order linear differential equations .... 919 Haim Hanani and Marian Reichaw-Reichbach, Some characterizations of a class of unavoidable compact sets in the game of Banach and Mazur .............

John Grover Harvey, III, Complete holomorphs . . . . . . . . . . . . . . . . 961 Joseph Hersch, Physical interpretation and strengthing of M. Protter's method for vibrating nonhomogeneous membranes; its analogue for Schrödinger's

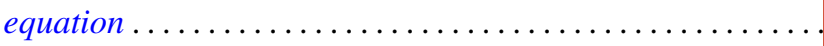

James Grady Horne, Jr., Real commutative semigroups on the plane...

Nai-Chao Hsu, The group of automorphisms of the holomorph of a group...

F. Burton Jones, The cyclic connectivity of plane continua

John Arnold Kalman, Continuity and convexity of projections and barycentric

coordinates in convex polyhedra

Samuel Karlin, Frank Proschan and Richard Eugene Barlow, Moment inequalities of

Pólya frequency functions .

Tilla Weinstein, Imbedding compact Riemann surfaces in 3-space. .

Azriel Lévy and Robert Lawson Vaught, Principles of partial reflection in the set

theories of Zermelo and Ackermann

Donald John Lewis, Two classes of Diophantine equations .....

Daniel C. Lewis, Reversible transformations . . .

Gerald Otis Losey and Hans Schneider, Group membership in rings and

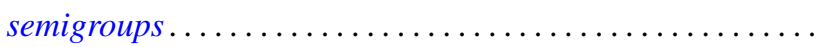

M. N. Mikhail and M. Nassif, On the difference and sum of basic sets of

polynomials.

Alex I. Rosenberg and Daniel Zelinsky, Automorphisms of separable algebras .

Robert Steinberg, Automorphisms of classical Lie algebras .... 